\title{
Up-scaling the island divertor along the W7-stellarator line
}

\author{
Y. Feng* \\ Max-Planck-Institut für Plasmaphysik, Euroatom association, D-17491 Greifswald
}

\begin{abstract}
Using the EMC3-Eirene code, the paper presents a numerical assessment of how differently the island divertor (ID) for the advanced low-shear W7-stellarators is expected to behave when enlarging the device from W7-AS to $\mathrm{W} 7-\mathrm{X}$ and further to a reactor-relevant size. It aims at identifying the potentially critical issues of the ID concept on up-scaling the machine size towards reactor, serving as a reference in preparing the divertor program starting soon on W7-X.
\end{abstract}

PACS: 52.55.Hc, 52.55.Rk, 52.40.Hf, 52.65.Pp

PSI-20 keywords: Island divertor, W7-X, Fusion reactor, Divertor plasma, Edge modeling *Corresponding author address: Wendelsteinstrasse 1, 17491 Greifswald, Germany

*Corresponding author E-mail: feng@ipp.mpg.de

Presenting author: Yuhe Feng

Presenting author e-mail: feng@ipp.mpg.de 


\section{Introduction}

The ID concept for the low-shear W7-stellarators utilizes the inherent resonant radial magnetic field components of the 3D shaped coils. The feasibility of the ID has been demonstrated on W7-AS [1, 2]. Its steady-state operation capability and reactor relevance will be explored on the much larger successor W7-X. Predictive studies of the W7-X ID have begun using the EMC3-EIRENE code [3, 4] in comparison with the W7-AS ID and the poloidal divertor of tokamaks as well [5]. Several essential changes in the ID transport behavior are expected from W7-AS to W7-X: A high-recycling recycling regime, which is absent from W7-AS, is predicted to exist in W7-X; The detachment regime in W7-X is shifted to lower separatrix density conditions; The intrinsic carbon impurity radiation distribution in detached plasmas, which exhibits a strong in/out asymmetry in W7-AS, will become more symmetric among the X-points in W7-X; Consequently, the total radiation fraction, which is clearly below $80 \%$ in W7-AS because of the asymmetric radiation distribution, can exceed $90 \%$ of the SOL power in $\mathrm{W} 7-\mathrm{X}$ - a level expected to be necessary in a reactor of the W7-family; Although the total recycling flux increases more than linearly with the area of the LCFS, the core penetration flux of the recycling neutrals and the neutral pressure in the divertor pump chamber do not scale with the device size, but remain more or less comparable with those in the much smaller W7-AS; There are also numerical indications of the possibility of slightly separating the power and particle channels by utilizing the specific island geometry to find a compromise solution between the particle and energy exhaust, which seems to be a critical point of the ID with growing island size. Furthermore, the absence of a transformer in W7-X presents a challenge in stabilizing the edge islands against plasma currents. All these have motivated a numerical examination of the reactor relevance of the W7-X ID. For this purpose, W7-X is linearly up-scaled by a factor of 4 and simulations have been made under reactorrelevant parameter conditions. The results will be presented and discussed in conjunction with W7-AS and W7-X. 


\section{Up-scaling of ID transport}

W7-X differs from W7-AS not only in the increased machine size but also in the upshifted operational $l$-window in which the $l=5 / 6,5 / 5,5 / 4$ island chains are suitable for the ID,

Table 1: major geometric parameters of W7-AS and W7-X. $L_{c}$ is the target-to-target connection length. $\Delta x$ and $\Delta y$ indicate the average radial and poloidal island width. For comparison, the last column estimates the ratio of the characteristic cross-field transport length to $\Delta y / 2$ for $D=1 \mathrm{~m}^{2} / \mathrm{s}$ and $C_{s}$ (sound speed) $=1 \cdot 10^{5} \mathrm{~m} / \mathrm{s}$.

\begin{tabular}{|l|c|c|r|r|c|c|c|}
\hline & $\mathrm{R}(\mathrm{m})$ & $\mathrm{a}(\mathrm{m})$ & $\mathrm{L}_{\mathrm{c}}(\mathrm{m})$ & $\mathrm{l}$ & $\Delta \mathrm{x}(\mathrm{cm})$ & $\Delta \mathrm{y}(\mathrm{cm})$ & $\left(\mathrm{DL}_{\mathrm{c}} / \mathrm{C}_{\mathrm{s}}\right)^{1 / 2} /(\Delta \mathrm{y} / 2)$ \\
\hline W7-AS & 2 & 0.12 & 100 & $5 / 9$ & 4 & 8 & 0.8 \\
\hline W7-X & 5.5 & 0.5 & 300 & $5 / 5$ & 8 & 60 & 0.2 \\
\hline
\end{tabular}

with $l=5 / 5$ being the standard case. The divertor-relevant geometric parameters for W7-AS and W7-X are listed in Table 1, where the connection length $L_{c}$ has only a representative meaning because of the complex 3D SOL structures and the high configuration flexibility. The radial size of the islands scales as $r_{i} \propto \sqrt{R b_{m n} / m \imath^{\prime}}\left(b_{m n}=\right.$ normalized resonant radial perturbation field, $m=$ poloidal mode number, $\iota^{\prime}=$ the shear) and the poloidal island size is given by $2 \pi a / m$ on average. Both the larger $R$ and smaller $m$ in W7-X contribute to enlarge the edge islands. The poloidal extension of the W7-X islands largely exceeds the characteristic perpendicular transport scales, avoiding cross-field momentum interaction between adjacent island fans, which is considered to the main reason for the absence of a high recycling regime in W7-AS reference [2]. This suggests that the SOL transport in W7-X must behave differently from that in W7-AS. This is indeed shown by EMC3-EIRENE simulations. Figure 1 compares the EMC3-EIRENE simulation results for W7-AS, W7-X and ASDEXUpgrade [5]. Impurity radiation is not taken into account, and the simulated plasmas correspond to an attached state without appreciable neutral-ion momentum loss. Aimed at configuration effects, same cross-field transport coefficients $\left(D=1 \mathrm{~m}^{2} / \mathrm{s}\right.$ and $\left.\chi_{\mathrm{e}, \mathrm{i}}=3 \mathrm{D}\right)$ are assumed for the three devices, while the power entering the SOL $\left(\mathrm{P}_{\mathrm{SOL}}\right)$ is linearly scaled with the area of the LCFS, assuming the values of 1,3, and $10 \mathrm{MW}$ for W7-AS, AUG and W7-X, respectively. The two curves for W7-X correspond to two cases with and without the control 
coils that can be used to fine-tune the internal pitch of field lines and thereby control the $\perp$-toII transport ratio in the islands. The resulting effects are clearly shown in figure 1 . With increasing control-coil current (Icc) and ensuing field-line pitch, the up-/downstream density correlation in W7-X approaches that predicted for the AUG tokamak. The high configuration flexibility of W7-X allows the divertor plasma to be controlled in a broad parameter range, thus bridging the gap between W7-AS and tokamaks.

The high sensitivity of the ID transport to the island structure demonstrated in figure 1 reveals two critical issues of the ID concept, i.e. the sensitivity of the edge islands against finite- $\beta$ effects and the reactor-relevance of the W7-X ID. The low shear in the W7stellarators favours large island formation. However, the resonance location is sensitive to perturbations induced by plasma currents because of the associated flat $\mathrm{l}$ profile [6]. The most critical one is the bootstrap current. In the absence of a transformer in W7-X, two strategies are being discussed for reducing the finite bootstrap current effects. First, current drive like ECCD can be applied to compensate the bootstrap current effects [7]. Second, specific configurations can be chosen that intrinsically have small bootstrap current, e.g. the high mirror configuration. The former is an active method, nevertheless facing the difficulties of controlling the detailed 1 profile in the confinement region and of low current drive efficiency in high density plasmas. The latter method is passive and obviously restricts the operational configuration range of the device. Here, for checking the reactor-relevance of the W7-X ID, we choose the high mirror configuration as an example and linearly scale it up by a factor of 4 to a reactor-relevant size [8]. For convenience, the virtual reactor is termed "W7-R" in the following. The high mirror divertor configuration is shown in figure 2. Without external perturbation fields the $5 / 5$ islands have closed flux surfaces where the field lines are opened by the targets. In order to reveal whether and how the ID transport changes from W7-X to W7-R, two respective density scans are performed using the same transport coefficients of D 
$=0.5 \mathrm{~m}^{2} / \mathrm{s}$ and $\chi_{\mathrm{e}, \mathrm{i}}=3 \mathrm{D}$. A $10 \mathrm{MW}$ SOL power is assumed for $\mathrm{W} 7-\mathrm{X}$ whereas $200 \mathrm{MW}$ is assumed for W7-R. In each scan the separatrix density is increased until the downstream electron temperature approaches $10 \mathrm{eV}$. Impurities are not taken into account. The results are shown in figure 3 where the downstream parameters are averaged over the target surfaces weighted by the thermal plasma pressure profile downstream. In both devices the development of the downstream plasma roughly follows the divertor plasma behaviour expected under high recycling conditions in tokamaks which is indicated by the dashed lines. In the high density range, the paths of the downstream densities depart slightly from the dashed line and tend to flatten. This is a consequence of the gradually reduced significance of parallel transport in favour of the perpendicular one and has been analysed in our pervious works (see e.g. [2] and [5]). The downstream density curve for W7-R lies significantly above the W7-X one. There are two reasons for this. First, the connection length is linearly scaled up with the machine size. Second, if the SOL power is scaled up with the surface, as it is roughly the case for the W7-X-W7-R comparison, the parallel energy flux density upstream increases because the power decay length scales weaker than linearly with $L_{c}$ (s. next section). Both raise the upstream temperatures and thereby the upstream plasma pressure and consequently the downstream density.

From the comparison results shown in figure 3 one sees a similar behaviour of the up/downstream plasma correlation in $\mathrm{W} 7-\mathrm{X}$ and $\mathrm{W} 7-\mathrm{R}$, except for the higher downstream densities for W7-R.

\section{Particle and energy exhaust}

Purely from a geometrical point of view, the total toroidal length of the strike lines potentially available from a separatrix bounded configuration is $2 \pi R 2 N$, where $N$ is the number of the $\mathrm{X}$ points existing in a divertor configuration. Low-shear stellarators have a large aspect ratio and a multi-X-point geometry. Unfortunately, this geometric potential cannot be fully utilized for 
power handling because of technical reasons. In this ideal case, the ID targets would have a helically closed form resonant to the $5 / 5$ island chain and the angle of incidence of magnetic field lines onto targets is limited by the small resonant perturbation field $b_{m n}$, which is typically of the order of $10^{-3}$. In order to increase the incidence angle up to a technically feasible level, the targets must be at least by one order of magnitude shortened and discontinuously formed, as they are in W7-X, which largely compensates the effects of large $R$ and $N$.

On the other hand, the long connection length of the ID does reduce of the peak power load. $\mathrm{L}_{\mathrm{c}}$ exceeds $1 \mathrm{~km}$ in $\mathrm{W} 7-\mathrm{R}$ which is almost one order of magnitude longer than that in a tokamak reactor of similar fusion power. Assuming the same perpendicular transport, EMC3Eirene simulations show a clear broadening of the power and particle channel from W7-X to W7-R, where $L_{c}$ increases by a factor of 4 . The profiles are taken poloidally along the vertical target at the toroidal position of maximum power load. The situation of plasma-surface interaction at this phi-plane is shown in figure 5, where the vertical target has its first contact with the plasma, while the horizontal target stands in the shadow of other target elements. The S-coordinate on the horizontal axis in figure 4 denotes the target distance relative to the strike point, pointing from the divertor gap (private region) away towards the island center (SOL region). Similar to tokamaks both the power and particle profiles exhibit much shorter decay lengths in the private region than in the SOL because of the large difference in $L_{c}$. The profiles change their slopes when approaching the O-point due to the specific geometry of the closed island. In the first decay region, the W7-R profiles are broader than $\mathrm{L}_{\mathrm{c}}{ }^{1 / 2}$-scaled from W7-X. This is attributed to the higher downstream densities established in W7-R (see figure 2). The particle profiles are shifted from the power deposition profiles towards the O-point, as a combined effect of the target shaping (see figure 5) and the long connection lengths in the islands. This shift increases with machine size and exceeds $10 \mathrm{~cm}$ in the reactor case. The clear separation of the particle channel from the energy channel in W7-R makes it less 
efficient to further lower the downstream temperatures by raising the plasma density, which is already reflected by the flattening of the target $T_{e}$ in the high density range shown in figure 3 . On the other hand, the existence of high particle fluxes outside the power channel is beneficial to particle exhaust. Thus, a compromise solution needs be found between particle and power exhaust.

\section{Radiative power removal under detachment conditions}

As shown in figure 4, a $200 \mathrm{MW}$ power results in a peak power load of $12 \mathrm{MW} / \mathrm{m}^{2}$ for $\mathrm{W} 7-\mathrm{R}$, already exceeding the $10 \mathrm{MW} / \mathrm{m}^{2}$ limit. In reality, much higher powers are expected to enter the SOL in a reactor [8]. For example, assuming a reactor of $3 \mathrm{GW}$ fusion power and ignoring the core radiation, the thermal power into the SOL is $600 \mathrm{MW}$. Linear scaling of the W7-R results would mean that more than $70 \%$ of the 600 MW power must be removed before reaching the targets. This power removal fraction increases to $85 \%$, if a factor-of- 2 margin is left to account for asymmetries in power load due either to technical uncertainties or physical effects, and increases further when taking neutron irradiation in reactor environment into account. For this reason, line radiation of light impurities is an intensively investigated concept. Nevertheless, this concept works only if the radiation layer can be controlled and kept away from the confinement core. In tokamaks, it has been shown that the X-point geometry favors impurity radiation and an intensive-radiation belt around the X-point, termed X-point MARFE, is usually observed in detached plasmas. The island chains in helical SOLs provide a multi-null divertor configuration where the number, location and geometry of the X-points can be adjusted externally, thus having the potential of more flexible control of the radiation location for an optimum power removal. Indeed, a geometric parameter window has been experimentally identified for W7-AS within which the radiation layer can be stabilized

outside the confinement region without loss of the global energy confinement [1]. Nevertheless, the stable detachment obtained in W7-AS is only partial. Hot spots are observed in detached plasmas at the strike points on the low field side. This is explained by EMC3- 
Eirene simulations to be a consequence of an in/out asymmetry in the carbon radiation distribution. A more detailed description of how the detached plasmas in W7-AS behave can be found in [9] and the references therein. The flux surfaces in W7-AS are compressed on the low field side and expended on the high field side. Carbon radiation prefers the X-points on the high field side because of the reduced perpendicular heat flux. Because of the asymmetric radiation distribution the total amount of radiation is limited to be less than $80 \%$ of the SOL power. A higher radiation fraction would mean a deep location of the radiation layer on closed flux surfaces. Figure 6 shows 3D simulation results for W7-AS and W7-X. In comparison with W7-AS, the flux surface geometry in W7-X is more symmetric. This is reflected by the poloidal distribution of the edge islands. In W7-AS 6 of the 9 islands reside on the high field side while the $5 \mathrm{~W} 7-\mathrm{X}$ islands are more evenly distributed over the LCFS. Consequently, the radiation distribution of the intrinsic carbon impurities becomes more symmetric over the $5 / 5$ island chain. More than $80 \%$ of the 10 MW SOL power is radiated by carbon immediately after detachment transition while the radiation layer stays still near the target. With further increasing plasma density, the radiation layer moves inwards and eventually approaches the X-points, while the total radiation power gradually increases and finally exceeds $90 \%$ of the input power (see figure 6).

\section{Summary}

The machine-size dependence of the ID performance has been examined using the EMC3Eirene code. Significant changes in the basic ID transport behavior are expected from W7-AS to $\mathrm{W} 7-\mathrm{X}$. The divertor islands in $\mathrm{W} 7-\mathrm{AS}$ are too small to separate the multiple parallel transport channels correlated with the fine island structures far enough from each other. As a consequence the W7-AS ID in many aspects behaves differently from the standard poloidal divertor of tokamaks. With larger islands, as in W7-X size, the up-/downstream plasma correlation approaches that in tokamaks. No significant changes in this correlation are identified on further increasing the device beyond $\mathrm{W} 7-\mathrm{X}$ to a reactor-relevant size, except for 
the target profiles. With fixed cross-field transport the power decay length increases faster than $\mathrm{L}_{\mathrm{c}}^{1 / 2}$-scaling. Linear up-scaling W7-X by a factor of 4 leads to a clear separation of the particle and power channels, raising the question of finding the optimum solution for plasma exhaust in a reactor. An estimate of the power load under more realistic reactor conditions by linearly scaling the first reactor simulation results indicates that most of the power entering the SOL needs to be removed from the power channel guided by field lines onto targets. Detachment simulations for W7-AS and W7-X yield typical radiation patterns that are closely correlated with the X-points of the edge islands. The carbon radiation in W7-AS prefers the inboard X-points due to the flux expansion on the high field side. The resulting asymmetric radiation distribution among the X-points strongly limits the radiative power removal capability of the W7-AS islands. In contrast, the flux surface geometry in W7-X is more in/out symmetric. Consequently, a more symmetric radiation distribution and, therefore, a higher radiation capability are predicted for the $\mathrm{W} 7-\mathrm{X}$ islands.

\section{References}

[1] P. Grigull et al, Plasma Phys. Control. Fusion 43 (2001) A175

[2] Y. Feng et al, Nucl. Fusion 46 (2006) 807

[3] Y. Feng et al, Contrib. Plasma Phys. 44 (2004) 57

[4] D. Reiter et al, Fusion Sci. Technol. 47 (2005) 172

[5] Y. Feng et al, Plasma Phys. Control. Fusion 53 (2011) 024009

[6] J. Geiger et al, Contrib. Plasma Phys. 50 (2010) 770-774

[7] Yu. Turkin et al, Fusion Science and Technology 50 (2006) 387-394

[8] C.D. Beidler et al, Nucl. Fusion 41 (2001) 1759

[9] Y. Feng et al, Nucl. Fusion 45 (2005) 89 


\section{Figure captions}

Figure 1: Comparison of divertor transport behavior in W7-AS, W7-X and ASDEX-Upgrade calculated by EMC3- EIRENE. $\mathrm{n}_{\mathrm{ed}}$ is the downstream density whereas $\mathrm{n}_{\mathrm{es}}$ represents the average density on the LCFS.

Figure 2: High mirror divertor configuration at an up-/down symmetric plane. Only the upper half is shown. The 5/5 island chain is opened by a vertical and horizontal target. The targets and the baffle plate form a divertor chamber for compressing neutrals, with a gap being left between the two targets.

Figure 3: Machine size dependence of up-/downstream plasma correlation - W7-X vs W7-R. The dashed lines indicate the scaling expected from two-point models assuming purely parallel transport.

Figure 4: Comparison of power and particle deposition profiles between W7-X (dashed, dot-dashed) and W7-R (solid) at a sparatrix density of $3 \times 10^{19} \mathrm{~m}^{-3}$. The lower picture shows the normalized profiles. The meaning of S-coordinate on the horizontal axis can be found in figure 5 .

Figure 5: Plasma-target interaction at the toroidal position of maximum power load in W7-X. For W7-R all the scales need to be multiplied by a factor of 4 .

Figure 6: Carbon radiation fraction as a function of the separatrix density predicted for W7-AS and W7-X. The separatrix density is varied in a range where radiation happens on open field lines. 


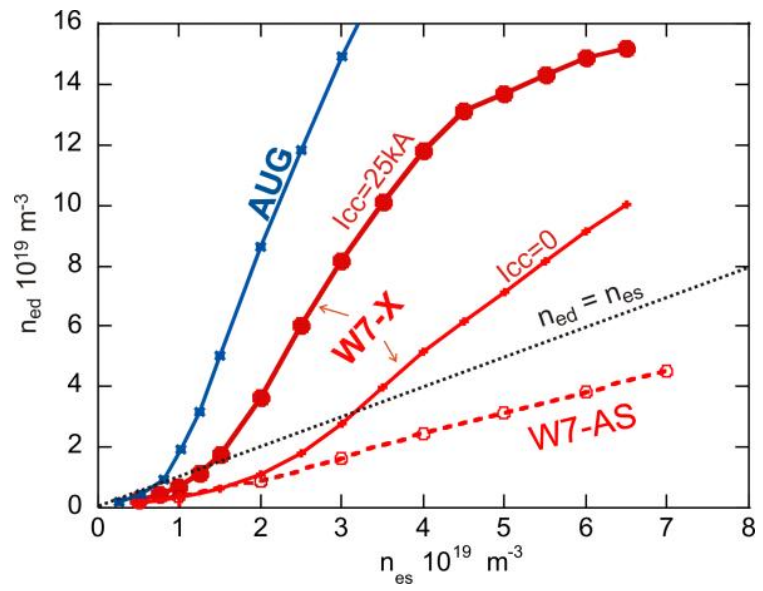

Figure 1 


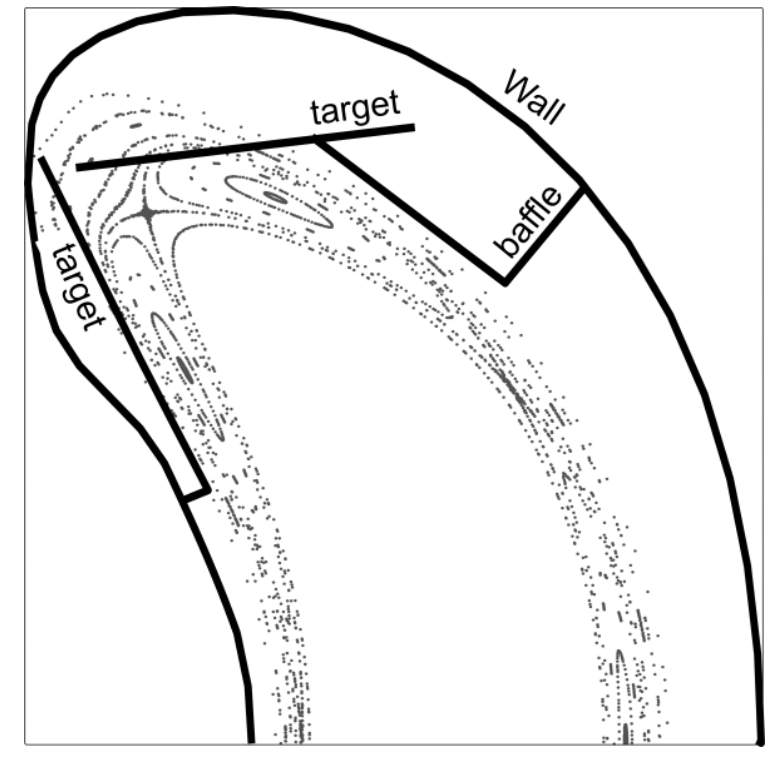

Figure 2 


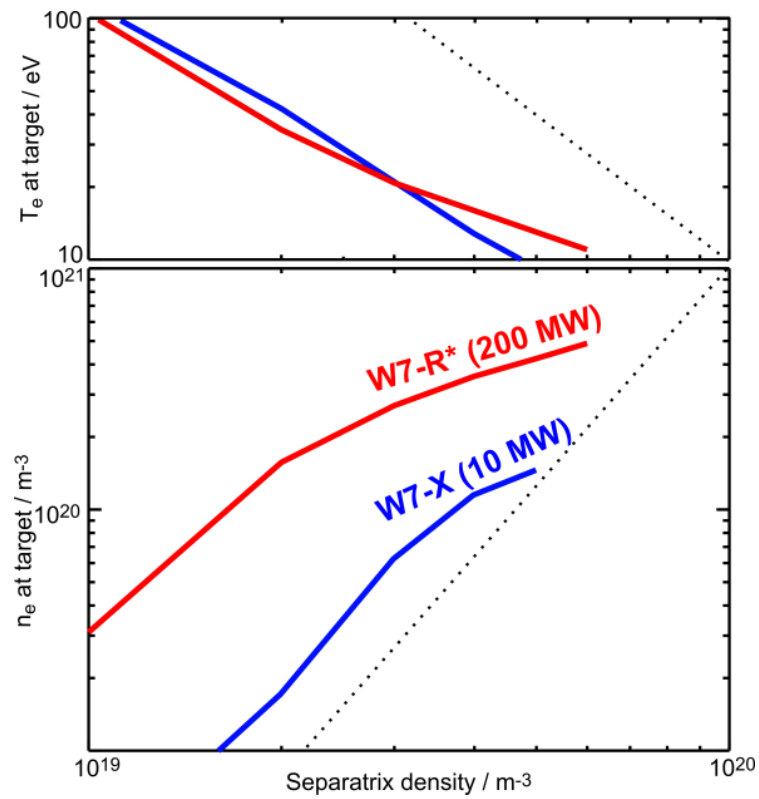

Figure 3 


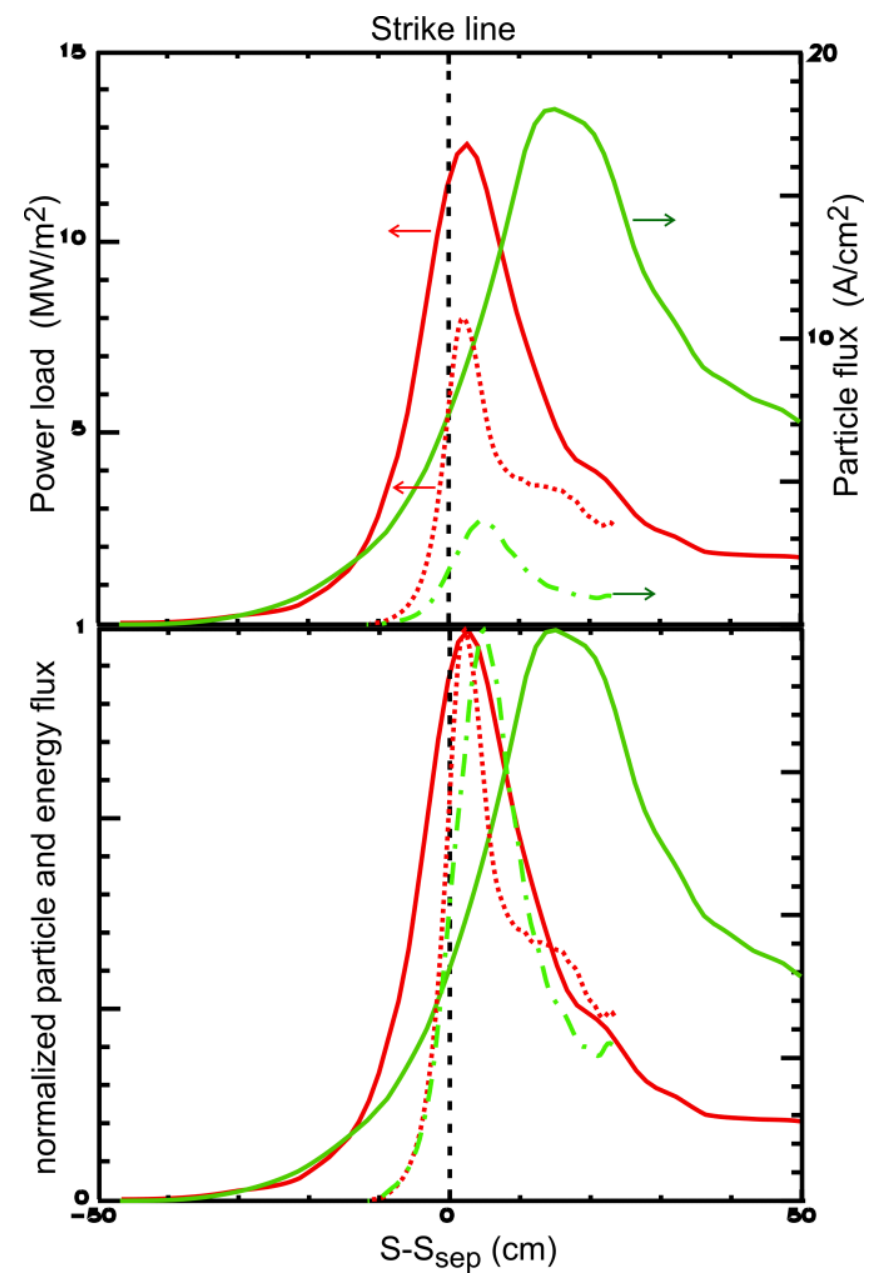

Figure 4 


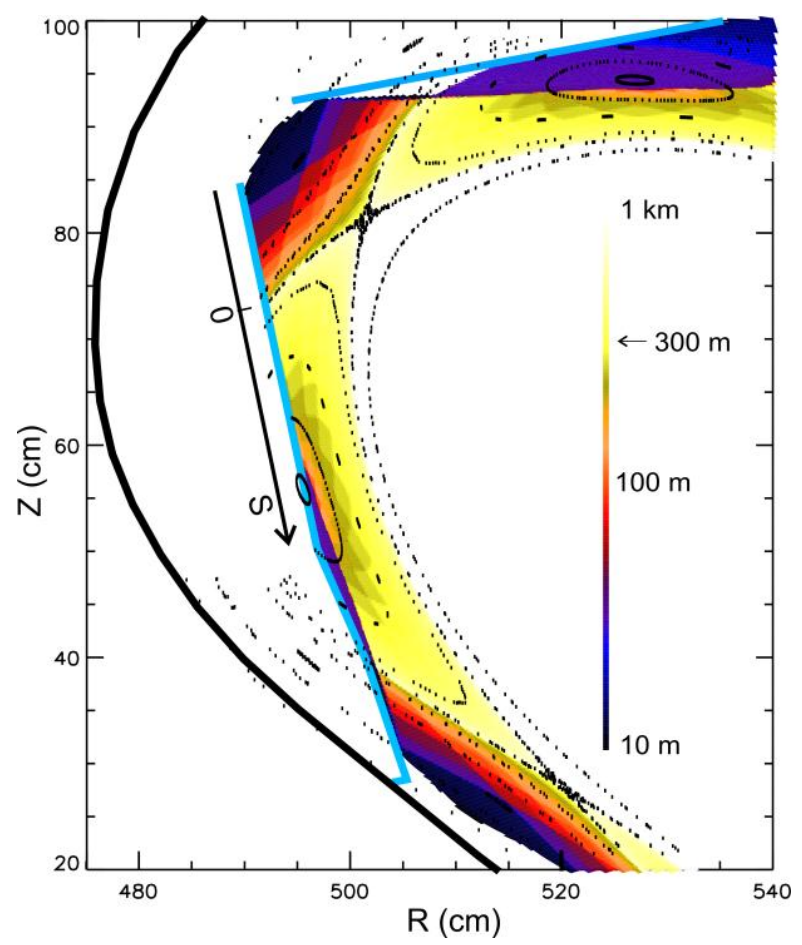

Figure 5 


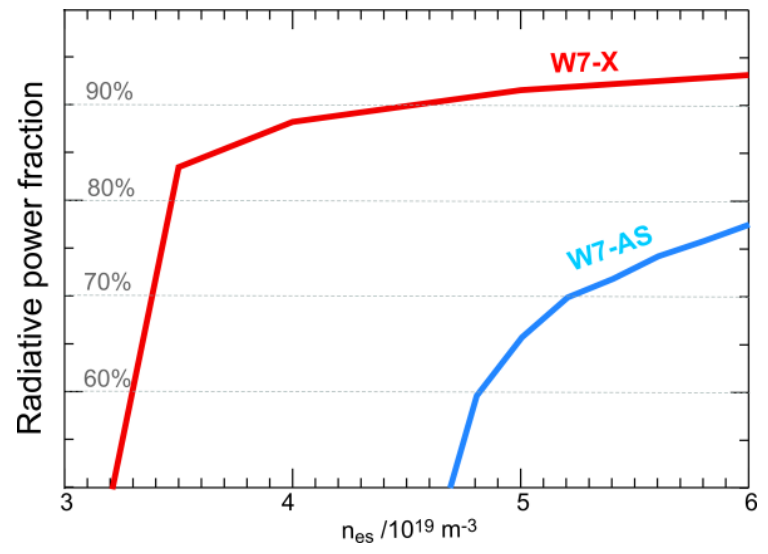

Figure 6 